\title{
Mercier de Compiègne et la question de la mode
}

RÉSUMÉ: Au XVIII siècle, la mode parisienne connaît de profondes mutations. L'offre vestimentaire variée est illustrée par une presse de mode devenue périodique qui renouvelle ses propositions grâce à de séduisantes gravures. Surtout une figure iconique émerge de ce siècle des Lumières : la marchande de mode et sa représentante la plus célèbre, mademoiselle Rose Bertin, «ministre des modes » de la reine de France, Marie-Antoinette. Les femmes étaient comme des oiseaux amusants qui changent de plumage deux ou trois fois par jour. Cet engouement pour la mode a provoqué de vives réactions notamment chez Mercier de Compiègne. Dans Comment m'habillerai-je ? Mercier établit un lien indéniable entre la mode vestimentaire et la puissance de la nation. Le respect de la simplicité traduit réserve et modestie; deux vertus caractéristiques de la nature humaine. Tout vêtement pouvant exalter les prétentions des femmes est en opposition avec leur destination naturelle. Les réflexions de Mercier sont emblématiques de ce retour à la nature. L'auteur de Comment m'habillerai-je ? exalte ainsi l'image de la beauté saine et naturelle. Ces réflexions veulent gagner également la Cour et changer les règles du bon goût.

MOTS-CLÉS : Mercier de Compiègne ; mode ; femme ; nature ; costume national.

\section{Mercier de Compiègne and the question of fashion}

ABSTRACT: The fashion in Paris at the XVIIIth century knows deep changes. The clothing offer is illustrated by a fashion periodic press which is renews its proposals thanks to attractive engravings. Especially an iconic figure emerges from this Age of Lumières: the fashion trader and his most famous representing, Miss Rose Bertin, "minister of fashion" of the queen of France, Marie-Antoinette. The women were as funny birds which change plumage two or three times a day. The women passion for fashion caused strong reactions, especially those of Mercier de Compiègne. In Comment m'habillerai-je ? Mercier established an undeniable link between the clothing fashion and the power of nation. The respect of simplicity, reflects reserve and modesty; two virtues characteristic of the human nature. Any clothing which can excite the claims of the women is against their natural destination. Mercier sings the return to nature. The author of Comment m'habillerai-je ? exalts the transparent and natural beauty image. These reflections want to allure the royal court and to change the rules of good taste.

KEYWORDS : Mercier de Compiègne ; fashion; woman; nature ; national costume.

RESUMO: No século XVIII, a moda Parisiense conhece profundas mutações. A oferta de roupa variada é ilustrada por uma imprensa de moda dita periódica que renova as propostas graças as gravuras atraentes uma figura icônica emerge deste século de Lumières : a comerciante de moda e a representante mais famosa dele: senhorita Rose Bertin, "Ministra das modas" da rainha de França, Maria Antonieta. As mulheres eram como pássaros engraçados que mudam a plumagem duas ou três vezes por dia. Esta admiração pela moda provocou reações profundas particularmente a Mercier de Compiègne. Em Comment m'habillerai-je? Mercier estabelece uma ligação inegável entre a moda vestimentária e

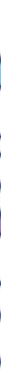


o poder da nação. O respeito pela simplicidade traduz-se reserva e modéstia; duas virtudes características da natureza humana. Qualquer artigo de vestuário que pode excitar as reivindicações das mulheres está em oposição ao seu destino natural. As reflexões de Mercier são emblemáticas deste retorno à natureza. $O$ autor de Comment m’habillerai-je? assim exalta assim a imagem da beleza, saudável e natural. Estas reflexões querem ganhar igualmente a Corte real e mudar as regras do gosto bom.

PALAVRAS-CHAVE : Mercier de Compiègne ; moda ; mulher ; natureza ; fantasia nacional.

La mode est l'affaire de goût et de distinction, d'apparences et de détails. Elle fonctionne en suivant un rythme propre et elle intervient quotidiennement dans la vie individuelle et collective. La distinction sociale qu'elle laisse transparaître est transcendée dans une forme de vie. La transformation de la mode est l'aspect le plus visible des mutations du corps social et le symptôme des nouveaux équilibres entre les individus. Ces changements sont évidemment réservés d'abord aux élites, mais au-delà des cercles étroits de l'aristocratie et de la bourgeoisie aisées et de leurs domestiques qui héritent des tenus portées par leurs maîtres, les citadins sont aussi influencés et l'opinion est confrontée à de nouvelles habitudes. L'introduction de nouveaux tissus - linon, mousseline - et l'enrichissement général choquent certains observateurs qui ne savent plus distinguer, dans la rue, la servante de la maîtresse. Le thème sert de motif à des réactions d'ordre pédagogique et politique, mais aussi comme projet de réforme pour Claude Mercier de Compiègne (1763-1800) dans son essai : Comment m'habillerai-je ? Réflexions politiques et philosophiques sur I'habillement français et sur la nécessité d'un costume national (1793). Mercier parle également du rôle de la femme dans la culture des apparences et sa position dans la société.

\section{La mode, une notion}

Le Trésor de la langue française (1985, XI, p. 917) définit la mode comme étant une « manière de se comporter propre à un groupe social, une région, un pays ». Le mot mode est synonyme de " costume, habitude, tradition ». Pour Le Grand Robert (1992, VI, p. 500), la mode signifie les « habitudes collectives et passagères en matière d'habillement ». II désigne comme synonymes : «mœurs, pratique, usage ». La définition du Littré (IV, p. 3928) est plus claire : La mode est un « usage passager qui dépend du goût et du caprice ». Robert en est inspiré du Dictionnaire de l'Académie française (1878, II, p. 217). En revanche, I'Oxford English Dictionary (I, p. 1341) utilise le mot français « mode » qui signifie «fashion »: " $A$ privailing fashion or costum, practice or style. A form, manner or variety ». Le Dictionnaire oxfordien garde le même sens donné par les dictionnaires français : "Conventional usage in dress, manners, habit of life, etc. ». En général, les dictionnaires attribuent à la mode un sens de comportement et d'usage passagers. C'est cependant ce dernier sens qui vient à l'esprit dès qu'on parle de la mode; ce changement constant des apparences vestimentaires que I'analyse critique suppose qu'elle le détermine.

La valeur de la mode s'inverse souvent par des rotations et des tournages réguliers des formes, qu'un trait d'originalité peut bientôt tourner en conformité et que la menace d'une indifférence généralisée la guette. Mais, c'est en cela qu'elle se trouve être un objet d'étude

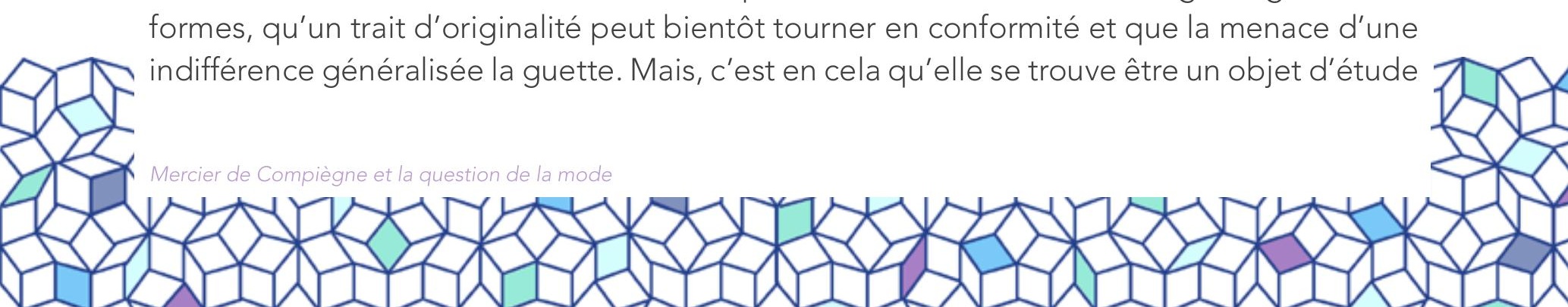


et demande à être traitée en respectant ses ambiguïtés. Les manières de s'habiller et le discours qui les entoure sont aussi des révélateurs de pratiques politiques et culturelles. L'allure vestimentaire est en ce sens marqueur d'identité culturelle et sociale. Par ailleurs, il convient de distinguer entre mode et discours sur la mode. Si la mode est étroitement liée à la variété des choix et des préférences individuels, le discours sur la mode est celui qui fixe le choix du vêtement. Mais dans la mode rien n'est plus parlant que l'image ; la description écrite de celle-ci n'est que support pour mieux la regarder. La mode ne se communique autrement que par l'image, elle est, pour ainsi dire, un langage visuel. Elle est placée sous le signe du concret et développe à elle seule un message: "Moi, je suis seulement la Beauté même. Je ne pense que par l'esprit de qui me contemple» (L'ISLE-ADAM, 1979, p. 74).

\section{Mode et rangs sociaux}

L'essai de Mercier est paru en 1793, I'année de la Terreur. Dans cette période transitoire de la Révolution, les idées et les modes de vie ont subi un changement considérable. Les crises politiques profondes ont conduit à la remise en cause du monde des apparences. La mode est conçue plus comme le reflet des mentalités et des mœurs que comme un art de vivre. Mode et politique ont toujours été liées l'une à l'autre. Pour exprimer une position politique, on utilisait les couleurs. La guerre des rubans et des cocardes au tout début de la Révolution le montre bien. L'égalitarisme révolutionnaire était poussé beaucoup plus loin en pensant que les Français devraient revêtir un uniforme. Salons et coteries, retrouvant leur lustre et leur pouvoir, font une grande partie de la vie politique avant et pendant la Révolution. Avant la Révolution, la mode était tout naturellement inspirée par la Cour. L'influence est donnée surtout par la reine, première dame élégante du royaume, et sa couturière madémoiselle Rose Bertin. Grâce au Cabinet des Modes, journal créé le 15 novembre 1785, les créations de Mlle Bertin sont diffusées dans toute l'Europe (SÉGUY, 1988, p. 40). Les magazines de mode, notamment La Galerie des Modes et Costume Français, qui apparaissent à l'époque de Marie-Antoinette, publient de très belles planches qui présentent des personnages en situation, avec toujours un petit commentaire qui précise les détails de la tenue portée. Cela donne : "petite maîtresse à la promenade », suivi d'un descriptif précis du tissu de la robe, du choix des dentelles, de la nature du chapeau. À cette époque, les femmes ne suivaient pas de «tendances » imposées. Elles faisaient réaliser leurs vêtements par une couturière selon des modèles qu'elles décidaient, s'inspirant souvent de la reine, la Cour, la noblesse. Le mot tendance (dans son acception d'aujourd'hui) est né avec le prêt-à-porter, quand la production de vêtements en série a réellement vu le jour.

L'apparition du coton et du lin avait influencé également le style de la fin du XVIII siècle. Avec une grande tendance, celle des robes-chemises en coton ou lin uni. Des robes qu'on enfile par la tête (avec toujours un corset dessous), très simples et très fluides, sans doute inspirées des femmes créoles qui préféraient porter des robes blanches sur lesquelles la transpiration se voyait moins. C'est Marie-Antoinette qui lancera la mode en 1783, avec un portrait officiel où elle se fera représenter par sa portraitiste favorite, Vigée Lebrun, dans une robe chemise. Le portrait fera scandale au sein de la cour : on considère que la reine est habillée de

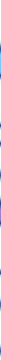


simples dessous, et elle sera obligée de poser à nouveau avec un vêtement en soie. Mais la mode est lancée et elle s'amplifiera encore durant le Directoire et le Premier Empire avec une profusion de robes blanches unies, s'inspirant cette fois de l'Antiquité gréco-romaine.

La mode de 1789 était éclectique et compliquée : robes à panier, chapeaux extravagants, etc. C'était une mode riche en tissus : draps d'or, soieries, damas, velours, brocarts, etc. Dès le XVIII ${ }^{e}$ siècle, l'innovation est constante et elle s'amplifiera encore durant le Directoire et le Premier Empire avec une profusion de robes blanches unies, s'inspirant cette fois de l'Antiquité gréco-romaine. La mode est à la couleur cheveux de la Reine, aux robes à la Levantine. Le bonnet Bonne maman, conçu par le Sieur Baulard, possédait un incroyable système rétractable, capable d'abaisser la coiffure en un clin d'oeil pour que sa propriétaire puisse se présenter d'une façon plus convenable, devant un religieux ou une personne d'âge respectable.

Après ces temps, on retourna à la simplicité. Les bourgeois étaient obligés de mettre au grenier leurs habits somptueux et de se vêtir comme les gens du peuple : caraco pour les femmes ${ }^{2}$, carmagnole pour les hommes ${ }^{3}$. Grâce à la Révolution, la simplicité fut au goût du jour. L'idée capitale était le retour à la simplicité naturelle: "Jamais une femme ne fut plus belle qu'avec ses cheveux nat[t]és, sans poudre et flottan[t]s au hasard sur les épaules » (MERCIER, 1793, p. 3).

L'année de 1790 voit l'amorce de cette période transitoire qui va aboutir à la transformation des lignes de la toilette. Pour les femmes, la tournure diminue de volume et remonte sur le haut des reins. La taille se marque encore à sa place mais les petits volets d'un corsage en rideau enveloppent la poitrine. Les manches longues dépassent le poignet et descendent sur la main jusqu'à la naissance des doigts. Les fichus, bouffant dans le décolleté, prennent le nom de fichus-menteurs, car ils peuvent tromper sur le volume réel de la gorge qu'ils voilent. La calotte des chapeaux s'étire en forme de pain de sucre sur une chevelure abondamment bouclée et sans poudre à laquelle Mercier fait allusion. Pour les hommes, les revers boutonnés de la lévite, en se déboutonnant pour se croiser sur la poitrine, donnent I'habit dégagé, avec un cran encore peu marqué à la naissance des basques, mais qui sera I'ancêtre du principal vêtement masculin du XIXe siècle.

Les événements ne sont pas favorables aux grands déploiements d'élégance et la noblesse cherche plutôt à passer inaperçue. Néanmoins, les membres du gouvernement, et principalement Robespierre, gardent une tenue soignée, tandis qu'au contraire les révolutionnaires du peuple adoptent pour uniforme et pour emblème le bonnet de lainage parfois bordé de devises ou de scènes allégoriques, la carmagnole portée à basque courte et le pantalon des matelots qui leur vaut le nom de sans-culottes. Dans les fêtes révolutionnaires, le blanc - symbole de pureté - est la couleur le plus souvent dévolue aux femmes et aux jeunes filles. Cette esthétique vestimentaire est orientée vers la simplicité, l'uniformité et le symbolisme politique. La simplicité, idée chère à Mercier comme à toute l'époque de la Révolution, est un sous-entendu de l'idée de nature ; une façon romantique de vivre,

\footnotetext{
Blouse droite à manches longues, portée flottante, c'est-à-dire sans ceintures sur une jupe froncée.
}

${ }^{3}$ Vestes à courtes basques, à grand collet avec plusieurs rangées de boutons de métal, portée d'abord dans le Midi par des ouvriers piémontais, originaires de Carmagnola. Elle fut introduite à Paris en 1792 par les fédérés marseillais et adoptée par les révolutionnaires. On la porta ensuite accompagnée d'un gilet tricolore et d'un bonnet rouge. 
dont les premiers éléments ont déjà existés chez Pascal, Montaigne et Rousseau. Pour ces philosophes, la nature traduit l'aspiration à un monde idéal et égalitaire. Rousseau la réduit à la simplicité, laquelle pendant la Révolution devient un mot d'ordre d'une évidence quasi intuitive et conçue comme une loi déterminée.

Quand on cherche la nature d'une chose, c'est l'ensemble de ses propriétés intrinsèques qu'on cherche. Cela dit, si la nature nous enseigne socialement la simplicité dans la façon de s'habiller, elle nous enseigne logiquement la manière de se connaître soi-même. De ce point de vue, la nature n'est pas seulement une façon de vivre, mais aussi une façon de penser, une vision du Monde et de l'Homme. C'est dans ce sens qu'au cours du XVIII siècle, on cherchait la différence entre les sexes, les aptitudes de la femme, la nature féminine, la femme naturelle; toute une littérature abondante sur ce qu'on peut appeler une physiologie naturelle. Le destin d'un sexe, en l'occurrence la femme, que révèle la nature, relève d'un choix à partir des tendances physiques et psychologiques qui fournissent des indications non pas tant sur une nature comprise comme donnée, que sur une nature saisie comme fin (HOFFMANN, 1977, p. 539). Mercier se déclare défenseur de cette vision :

La misère va donc opérer chez nous ce que n'aurait jamais fait la raison, et la cherté progressive de toutes les marchandises va nous ramener bientôt à la plus grande simplicité. $(1793$, p. 3)

Une condamnation totale de la civilisation oblige selon Mercier à se retourner à la simplicité. C'est dire que le discours moralisant l'emporte sur la dimension esthétique puisque la façon de s'habiller révèle les mœurs de la société.

\section{La mode et l'identité sociale}

Après avoir rappelé qu'un habit n'est pas seulement une protection, une marque de pudeur et une ornementation, Roland Barthes (1957, p. 432) critique l'absence de réflexion et de définition sur ce que pourrait être, à un moment donné, un système vestimentaire et l'ensemble axiologique qui le constitue, à savoir contraintes, interdictions, tolérances, aberrations, fantaisies, congruences, exclusions. Barthes suggère de comprendre le vêtement dans son contenu idéologique comme un langage suivant le modèle saussurien et d'analyser ensuite comment le costume s'insère dans un système normatif bien organisé. Les signes de la mode sont bien incorporés et investi par un sujet, exactement comme il en est des signes linguistiques. Ainsi il distingue entre vêtement et costume. Le vêtement relève d'un choix individuel, tandis que le costume appartient à une norme collective. Par le vêtement, dit-il, "I'individu actualise sur lui l'institution du costume »(BARTHES, 1957, p. 432). Selon Devocelle (1989, p. 83), le vêtement avait pendant la période révolutionnaire une fonction politique ou didactique. On utilisait le vêtement pour montrer certains principes idéologiques. On essayait d'étendre le costume uniforme à toutes les classes sociales, et certaines couleurs étaient utilisées comme signes politiques. Mais pour les paysans et les artisans, le vêtement 
présente avant tout un rôle fonctionnel. Pour la noblesse et la bourgeoisie, il est une marque de distinction, I'affirmation d'appartenance à une communauté et de s'y reconnaître.

Dans un conflit Monde ancien/Monde nouveau, le changement du régime ne pouvait laisser subsister des distinctions caractéristiques des ordres privilégiés et par conséquent de la monarchie absolue. Les députés se trouvèrent confrontés à des difficultés idéologiques pour appliquer au costume les droits nouvellement proclamés : égalité et liberté, uniformité et diversité, en en étant les projections immédiates. En effet, dès la convocation des États Généraux, le costume de cérémonie prescrit aux députés des trois ordres fut contesté. C'était principalement le fait des élus du Tiers État. Ils reprochaient à la noblesse de se distinguer par la richesse de son habit à parements et boutons d'or, cravate de dentelle, et par son chapeau à panache blanc dit à la Henri IV.

Le costume a acquis d'emblée une valeur juridique alors que l'on revendiquait l'égalité politique entre les députés. Jean-Baptiste Salaville, journaliste et écrivain politique proche de Mirabeau, alla même jusqu'à proposer un costume uniforme aux trois ordres afin de répondre précisément et visuellement au droit d'égalité. II écrivit dans une lettre du 10 mai 1789 :

Les membres qui la composent (l'Assemblée) ne devant point se regarder comme députés de tel ou tel ordre, mais comme les vrais représentants de l'universalité du royaume, ne peuvent dispenser de voter l'uniformité du costume [...] symbole de cette égalité de droit et de pouvoir dont ils sont essentiellement revêtus. (Cf., DEVOCELLE, 1989, p. 99)

Dans Les Nuits de Paris [1788-94], Rétif de la Bretonne (1978, p. 234), rappelle aussi le renoncement au luxe vestimentaire et la promotion d'un "uniforme civique », simplifié et modeste, sinon populaire. Selon Devocelle (1989, p. 84), en cette période libérale de la Révolution, ces positions furent isolées. Après la chute de la monarchie, mais surtout à la suite de la proclamation de la République le 22 septembre 1792 (I'an I), le discours se radicalisa. On voulait réformer le costume dans ses aspects aussi bien civils que politiques. L'esprit de I'an II affirma pour le costume le rôle de symbole et d'emblème d'une République où tout était à recréer. Le symbolisme politique et juridique du vêtement dépasse à cette date l'emploi des couleurs nationales. Le vêtement n'est plus attaché à une condition sociale, mais devient signe politique et juridique. Cette nécessité conduira à empêcher toute possibilité de dissimulation. Les événements obligent à la transparence et le vêtement prend l'allure uniforme. La régénération du costume devint donc le thème principal de certains projets de constitution, mais aussi des écrivains comme Mercier des années 1793-1794. Au moment même où Mercier rédigeait son essai sur le costume national, Jean-François Barailon, député du département de la Creuse, présentait son projet et énonçait la nécessité d'un costume unique pour tous les citoyens, enfants de la patrie et soldats ${ }^{4}$.

C'est le principe d'égalité qui fondait le projet, mais Barailon distingue entre l'habit de la ville et I'habit politique. Cette régénération s'inscrivait dans la représentation d'une cité idéale, militaire où l'homme devait être distingué par les couleurs de son habit. Pour la

Projet de constitution présenté à la Convention nationale le 1e juin 1793 par J-F. Barailon. Cf., Archives parlementaires, t. 67, p. 220, 24 juin 1793, annexe XIV. Les articles 51-52 section I, et 1 à 5 section XXIV sont consacrés exclusivement au costume national.

Mercier de Compiègne et la question de la mode 
première fois on parlait d'un costume national, mais également des costumes obligatoires et différenciés pour tous les mandataires de la cité. Le même intérêt pour le costume national a été annoncé par Mercier pour contrecarrer le projet de David. Comment m'habillerai-je ? est un essai qui fait partie du débat tout aussi fervent et passionné sur la manière de s'habiller. Son objectif est de faire du costume uniforme le moyen de distinction entre les rangs sociaux. L'idée remonte à Platon qui avait séparé, jusque dans leurs habits, entre la classe gouvernante, la gardienne et l'ouvrière. Le même principe existait dans les sociétés romaine et grecque. Ainsi pour Mercier, le vêtement est un support d'identité sociale : " Je crois qu'il n'y aur[a]it pas d'inconvénient à ce que chaque état soit distingué par une forme d'habit et d'une couleur particulière » $(1793$, p. 4). Mercier tire sa position d'une scène particulière, quand il se rendit chez une duchesse où il ne sut pas distinguer entre elle et la femme de chambre. La scène pourrait être une plaisanterie féminine, mais le jeune poète fut sérieux et décida de réagir contre un vêtement qui n'offre aucune « différence sensible » $(1793$, p. 6) entre les gens d'en haut et les gens d'en bas.

L'objectif de Mercier est de démontrer, dans son projet philosophique, la nécessité « de mettre une différence dans les costumes et empêcher qu'on ne prenne désormais une soubrette pour une duchesse et le valet de chambre, décrassé et habillé, pour un grand seigneur » $(1793$, p. 8). La mode doit donc suivre ce que la nature a déjà établi de différence entre les rangs sociaux. II serait contrenature qu'une servante soit habillée comme une maîtresse. Le rôle fonctionnel du vêtement est indissociable de son rôle signifiant. La façon de s'habiller doit être en harmonie avec les règles naturelles. Malgré le partage du même principe de la raison naturelle, source de fraternité et d'égalité, Mercier prend une large distance par rapport à l'esprit de la Révolution et préconise un costume différent pour chaque rang social. Deux ans avant la publication de Comment m'habillerai-je ? le 30 juillet 1791, I'Assemblée supprima toutes les décorations propres aux ordres de chevalerie et aux corporations et tout signe extérieur supposant des distinctions de naissance.

Par ailleurs, l'intérêt pour le costume national uniforme était présent dans les débats de la Société populaire et républicaine des arts. La Société a travaillé sur un projet de concours visant à régénérer le vêtement utilisé alors dans la société. L'artiste précurseur de ce mouvement d'innovation était considéré comme le seul apte à ramener l'art sous l'autorité de la raison. II s'agit bien de David auquel Mercier fait allusion dans son "petit mot d'avis ». Mercier insiste d'avantage sur le respect des rangs sociaux dans un contexte révolutionnaire défavorable politiquement et juridiquement. Le terme « rang » est fréquemment utilisé pendant l'Ancien régime. On le substituait pendant la Révolution par le terme «fonctionnaire ». Il va de soi qu'un fonctionnaire est un citoyen de la patrie.

Le fruit des réflexions des artistes républicains de la Société populaire et républicaine des arts est une plaquette intitulée "Considérations sur la nécessité de changer le costume français» (DELAPIERRE, 1996, p. 152). La Société propose que le costume respecte la liberté sous toutes ses formes, il doit encore respecter l'égalité totale, ne distinguer ni les fortunes ni les rangs, donc être le même pour tous les citoyens, militaires compris. Malgré la même référence à l'Antiquité, Mercier prend une attitude distanciée à l'égard du projet

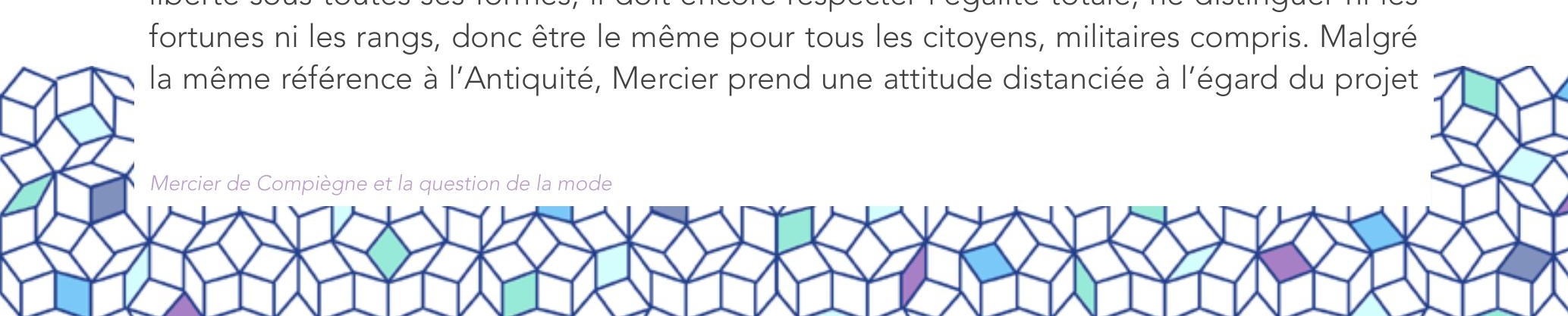


de la Société. Pour celle-ci, l'Antiquité est une source de réflexion artistique et politique permettant de faire de la liberté un principe fondamental. C'est ainsi que l'on finit par proposer un costume unique, propre à gommer toute inégalité dans la plaquette que l'on a mentionnée et cela à partir de 1793-1794. Or le recours de Mercier à l'Antiquité est plutôt moral : les Germains sont le modèle à suivre pour leur respect des rangs sociaux et pour la valeur de leur goût vestimentaire.

\section{Les Lumières et la femme}

La Révolution est une époque où la mode avait tendance à rechercher l'apparence naturelle. L'appel de Mercier à la simplicité permet au corps féminin de se débarrasser de tout accessoire destiné à le modeler et de retrouver par la suite ses formes et sa souplesse naturelles. Tandis que le projet de la Révolution d'un costume national est inspiré de l'égalité de tous les citoyens, Mercier préconise un costume national qui ne mettra pas en cause les inégalités des classes. II garde cependant l'idée selon laquelle le luxe est la prérogative des femmes, un art propre à elles. La femme aime se plaire aux yeux des autres. Encore faut-il aider son physique à s'accomplir dans ce sens. C'est ce physique qui attire l'attention et le désir. Mercier ramène ce comportement au libertinage (1793, p. 12). Ce terme dont le sens péjoratif est évident, nous rappelle combien la femme est responsable de la surabondance des objets de luxe et de fantaisie.

Or les superfluités qu'enfantent la fantaisie et le luxe sont la cause de la décadence morale et des crises économiques. Selon Mercier, par rapport au poids du luxe, " les hommes sont devenus petits » (1793, p. 8). La marchandise de luxe produit la fausse richesse, beaucoup plus de fripons que de sages. Si le luxe est par définition un mode de vie caractérisé par de grandes dépenses consacrées à l'acquisition de biens superflus, par goût de l'ostentation et du plus grand bien-être (Dic. Robert), il est naturellement l'œuvre des dames. Nous pouvons comprendre pourquoi Mercier «supplie » les femmes d'être naturelles : "Eh, de grâce, laissez à la nature le soin d'arranger vos cheveux » (1793, p. 6). Cet engouement pour les apparences est chez les femmes un signe de faiblesse et de légèreté auxquelles I'homme doit prêter attention.

Le rapport entre la faiblesse de la femme et l'attirance pour le luxe est bien une idée inscrite dans le discours naturaliste des médecins des Lumières. Dans ce discours, morale et biologie se parlent et se répondent à I'unisson (PERROT, 1984, p. 157). La femme est passée ainsi de l'autorité du prêtre à celle du médecin. L'un des défenseurs de cette position est le docteur Pierre Roussel, auteur de Système physique et moral de la femme publié en 1775. C'est Roussel l'inventeur de la «nature féminine » (KNIBIEHLER, 2003, p. 128). L'anatomie féminine insiste sur deux traits : la faiblesse et la prédestination à la maternité. Le discours médical, renforcé par la mise en place d'institutions comme la Société royale de médecine, justifie l'infériorité de la femme, "la femelle de l'homme » comme le dit Diderot. Les arguments ne sont plus théologiques, mais «scientifiques».

Qu'ils soient philosophes contestataires comme Diderot ou académiciens comme Thomas ou médecins comme Roussel, Pinel et Virey, presque tous se retrouvent dans la conviction que

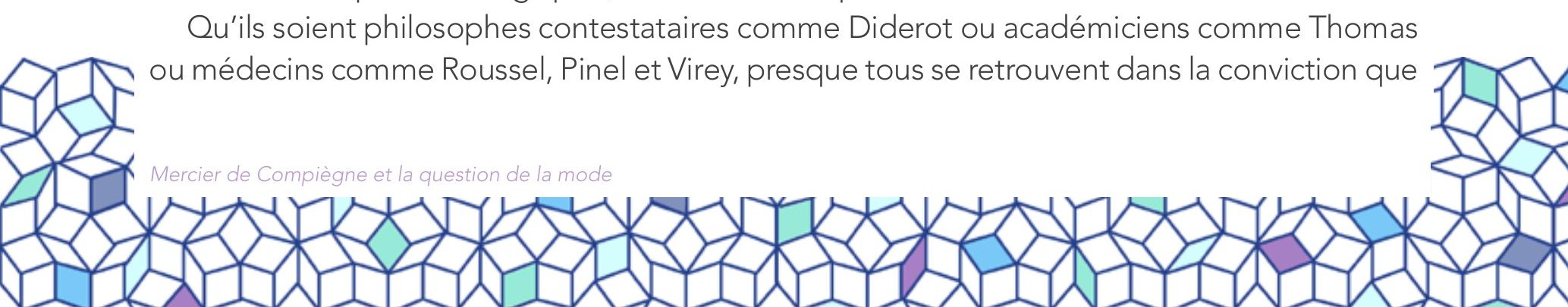


la femme se résume à sa matrice, source d'émotions incontrôlées pour les hommes bien entendu. L'anatomie conclut que la femme est faible par son sexe, son corps et son âme. Toutes ces facultés laissent entrevoir "l'état passif », dit Roussel, auquel la nature destine la femme. Eternelle enfant, soumise à la « nature », versatile, charmante et dangereuse, la femme appartient bien à l'universalité du genre humain, mais sur un mode inférieur, " passif ». La vieille division entre I'homme chaleureux, solaire, ferme, tourné vers l'extérieur, et la femme froide, lunaire, aquatique, vouée à l'intérieur - exactement l'inverse de l'homme - se prolonge via les nouvelles connaissances distinguant entre l'homme cérébral et la femme utérine.

Nul ne pense contre son corps, la femme encore moins que l'homme. En effet, l'anatomie physiologique étudie aussi les facultés intellectuelles. La femme a une plus grande richesse, une plus grande variété de sensations (CLINTON, 1975, p. 287). II pourrait s'ensuivre une vitalité remarquable de l'intelligence, puisqu'on reconnaît depuis Locke et Condillac que la sensation est le point de départ de la pensée. Mais deux facteurs bloquent l'essor des facultés mentales féminines : les facteurs organiques et les facteurs sociaux. D'une part, les sensations internes exercent une action puissante sur le comportement. Or chez la femme, il existe une densité d'innervation dans les organes génitaux. L'extrême sensibilité de ces organes influence constamment les glandes, les humeurs, le cerveau. D'autre part, la femme est engagée dans la vie sociale d'une toute autre manière que l'homme. Assaillie en permanence et de tous côtés, elle ne peut parvenir à une maturation suffisante des idées, encore moins à une pensée déductive profonde. La concentration et la réflexion lui sont impossibles.

Selon le docteur Virey, la femme souffre d'une "frivolité de goût », d'une "versatilité éternelle d'idées et de penchants » qui retentissent sur toute sa conduite (KNIBIEHLER, 2003, p. 134). Pour le docteur Roussel, « la science que les hommes achètent presque toujours aux dépens de leur santé » n'est pour les femmes qu'une «vaine fumée d'orgueil » qui leur donne des vapeurs et de l'hypocondrie (KNIBIEHLER, 2003, p. 134). Le raisonnement de ce discours « scientifique » est très cohérent au point que Napoléon dira plus tard : " L'anatomie est le destin ». C'est bien ce discours qu'inspire à Mercier une anatomie sociale du costume. Mercier redoute que la société ne se féminise, notamment dans des classes élevées, celles qui sont assez riches pour acheter les produits de luxe:

Plus les règnes ont offert à la France de grandes choses et de révolutions importantes, moins le luxe des habits a eu de vogue, tandis qu'au contraire, plus il ont été faibles ou transitoires, plus le luxe a triomphé, plus on a vu de disparition dans les finances, d'inégalité dans la fortune des particuliers, de tyrannie dans les fermiers généraux, de gens d'affaires ruinés et de mendian[t]s. (MERCIER, 1793, p. 12).

Mercier soutient l'idée selon laquelle la pauvreté a pour cause la propagation du luxe. Il s'ensuit que la mode féminine est un signe de faiblesse politique et économique. II faut, seIon Mercier, rationaliser le choix d'habillement pour atteindre une telle simplicité naturelle. La réforme doit commencer par la forme extérieure avant d'arriver aux goûts : " II faut aller pas à pas pour gagner quelques choses » (1793, p. 13). Mercier demande aux citoyens de

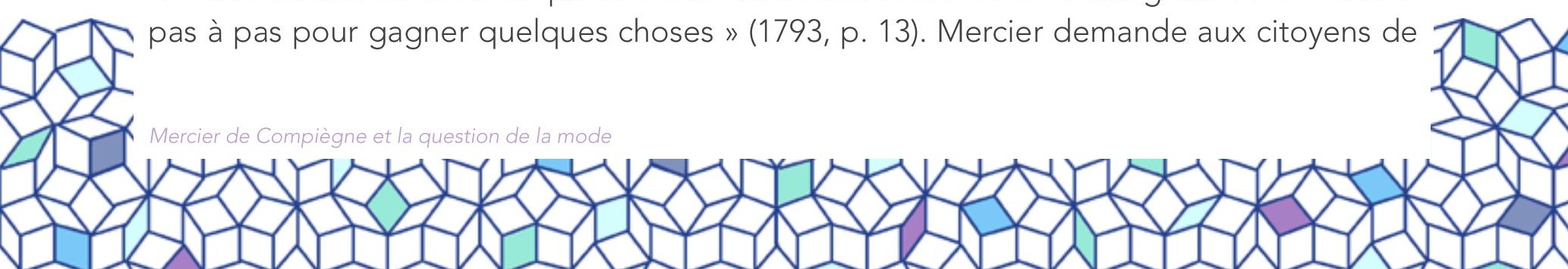


" se rapprocher de la nature » (1793, p. 13). L'habit doit avoir " une valeur réelle et permanente » (1793, p. 14) de la personne qu'il porte. II doit refléter en ceci la forme et le contenu, l'extérieur et l'intérieur, le dedans et le dehors de la personne. Le modèle est Rousseau malgré son habit à l'arménienne. La forme doit être commode, c'est-à-dire en harmonie avec la taille physique et la valeur sociale de l'homme :

Je voudrais que chaque classe d'artisans et de citoyens bourgeois adoptât un costume uniforme qui réunit l'agréable et l'utile, la richesse sans luxe, le goût sans ridicule, la nature sans indécence, et le plus grand avantage pour la santé. (MERCIER, 1793, p. 14)

Il faut garder la pudeur, selon Mercier, il ne faut pas blesser « l'austère décence » qui est une des bases de la "valeur réelle » du costume de l'Antiquité. Le costume ne doit pas donc avoir une connotation sexuelle. Il doit par conséquent être «large et volumineux ». Le costume est une représentation esthétique, mais surtout identitaire ; il reflète l'identité de la personne, sa valeur profonde en cachant les traits physiques. C'est donc le corps qui est en question ici. Enfouir le corps sous l'habit ne suffit pas à l'exclure, au contraire le regard de chacun sur l'autre rappelle à tous leurs défauts et leur contingence. Le corps est menacé par l'intempérance vestimentaire. Mercier éprouve une nostalgie de la modestie et de la spontanéité ; signes de joie et de simplicité.

À l'époque de Mercier, la vie publique était objet d'études, d'analyses et d'interprétations compulsives. L'intimité, la familiarité, le relâchement des apparences confrontent des êtres "démaquillés », libérés des « affaires » et des codes extérieurs, fascinés par la transparence et la fusion (PERROT, 1984, p. 142). Cet état a produit une double tyrannie : celle de la sincérité personnelle et de l'artifice public. Mercier demande alors une plus grande maîtrise de soi. C'est une vertu naturelle de paraître sincère et transparent, à laquelle l'apparence vestimentaire doit correspondre. Le thème de la mode s'inscrit dans un projet global pour la réforme des mœurs. II dénonce ainsi le luxe mauvais, l'égalitarisme révolutionnaire, la convoitise féminine, le « libéralisme » de la mode, tout en proposant à chaque classe d'artisans un costume uniforme. Le vêtement commode et simple que Mercier propose a pour objectif, I'hygiène collective et la réduction de la consommation. La dimension collective de la mode favorise l'égalité, tandis que la dimension morale distingue entre les personnes relativement à leur âge, sexe, condition sociale.

Puisque la littérature fixe les manières de vivre, Mercier recourt à l'utopie de son époque, notamment au Voyage autour du monde (1771-1772) de Louis Antoine de Bougainville (17291811). II recommande aux femmes d'imiter la belle et simple othaïtienne de Bougainville (MERCIER, 1793, p. 15). Ainsi Mercier ne résiste pas à l'envie de s'inspirer de la pensée utopique qui caractérise I'an I. Le costume, dans cette cité nouvelle, est un instrument d'éducation permanente, apte à diffuser et à enseigner les nouvelles valeurs. Ceci montre que les réflexions de l'auteur s'inscrivent également dans le vaste projet éducatif de l'époque. Elles brassent des interrogations analogues et proposent des solutions comparables.

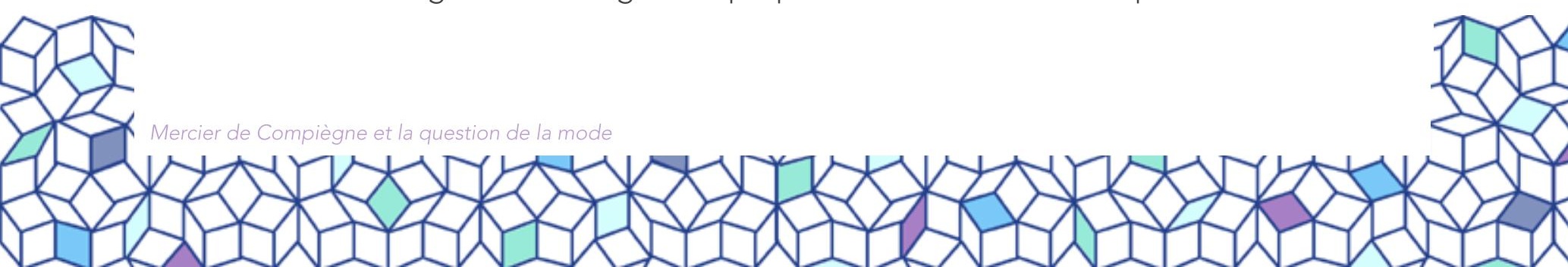




\section{Langage de la mode}

Comment m'habillerai-je ? Dans cet essai, Mercier met l'accent sur « l'étrange idiome associé à notre langue par les délires du luxe » $(1793$, p. 8). S'il est un domaine où se déploie I'incroyable variété des termes, c'est bien celui de la coiffure entre 1774 et 1790 (VITTU, 1989, p. 41). Selon Mercier (1793, p. 8), la variété des termes est dans toutes les variations des fantaisies : les meubles, les coiffures, les habits, les ragoûts et les voitures. II faut pour les connaître se fier aux gravures et publications de l'époque. Le document le plus intéressant à cet égard est le premier journal de la mode française. II parut sous trois titres successifs : Cabinet des modes (première année), Magasin des modes nouvelles (deuxième à quatrième année) et Journal de la mode et du goût (les trois dernières années), de 1785 à 1793. Le journal partage le même souci que Mercier, à savoir le danger que représentent les débordements en tout genre.

La richesse du vocabulaire des vêtements nous montre qu'il existe une véritable rhétorique de la mode. Le vocabulaire montre bien que la mode s'est institutionnalisée. Par ailleurs, le discours sur la mode a eu une grande influence sur les choix et les comportements. Ainsi les modèles, préconisés par Mercier, nous montrent-ils des dames ayant l'air grave et résolu, comparés aux modèles pré-révolutionnaires qui riaient, s'adonnaient au jeu et jouissaient d'une insouciance apparente (KLEINERT 1989, p. 69).

S'il existe un riche lexique, c'est parce qu'il existe une variation des vêtements et accessoires. Mercier cite "les termes essentiels» $(1793$, p. 8) du vocabulaire de la mode. Les exemples varient entre les vêtements (l'Ottomane, Frac, Caraco, Steinkerque), les voitures (Berline, Phaëton, Turgotine), l'eau-de-vie (Whiski), les chapeaux féminins (Cabriolet, la désobligeante), les bonnets (Cagliostro, la grenade, Malbrough), les tabatières (Turgot) et d'autres termes de vêtements comme mirliflore, chenille, pet-en-l'air, polonaise, nierrot, lévite, fichu, etc. (MERCIER, 1793, p. 8-11). Etymologiquement, il y a des termes qui renvoient à des moments de gloire : batailles, villes prises par des princes guerriers, etc. Mercier dénonce la transposition d'une terminologie de guerre à un champ lexical de luxe et de bien-être. Le vocabulaire de la mode n'est donc pas approprié ; il est de caractère passager tout comme l'objet sur lequel il porte. Par exemple la Grenade est une ville qui fut prise au Moyen âge, mais «chez nous, c'est un bonnet » (MERCIER, 1793, p. 9). Quand on parle de la ville, le mot «Grenade » est approprié, contrairement à son utilisation quand on parle du bonnet. Ceci montre que la mode ne dispose pas d'un vocabulaire original. Elle ne semble point, dans ce cas, faire partie de l'intérêt de la société. De surcroît, si la société témoigne d'un intérêt pour la mode, elle montre par là un signe de faiblesse.

Qui sont les " coupables" ? Mercier accuse en premier les artistes. Ceux-ci, grâce à leur imaginaire, "ont trouvé le secret d'exciter l'attention et le désir, en faisant tenir les modes aux événements publics » $(1793$, p. 9). L'histoire de la guerre s'est transformée en boutons, boucles et tabatières. De l'autre côté, les femmes élégantes, cédant à leurs caprices, ont beaucoup encouragé la mode et ont beaucoup influencé les hommes politiques et les citoyens ordinaires. Les femmes sont les juges dans le domaine de l'apparence. La

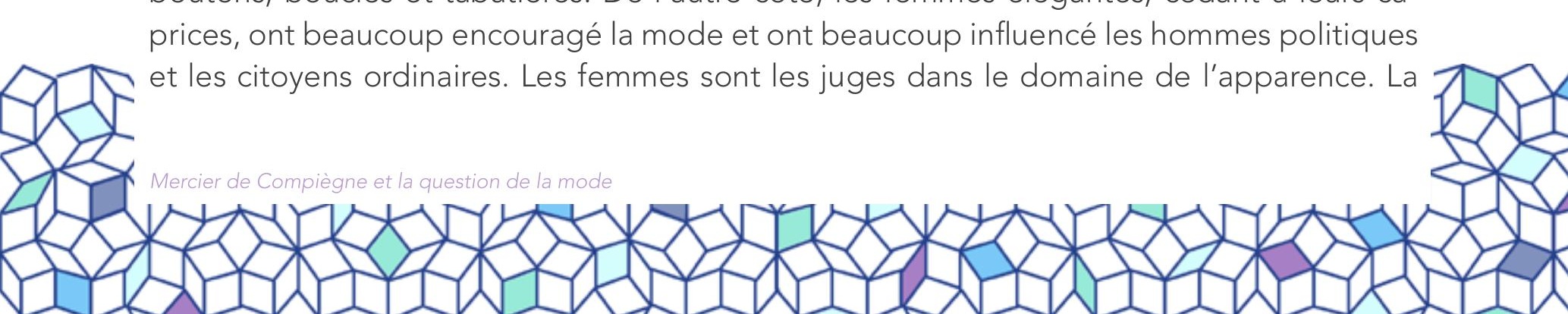


mode est le domaine où la femme l'emporte sur l'homme. La terminologie de la mode ne semble alors pas être spontanée. Aussi la mode est-elle un ensemble d'objets tous destinés à l'usage des femmes. C'est dans la mode que la femme prend sa revanche sur l'homme. Elle peut ainsi dire par exemple : "Vous avez votre Grenade, nous avons aussi la nôtre ». Par conséquent, la terminologie ne renvoie pas seulement à une transposition des évènements historiques, mais bien aussi à un comportement féminin. Puisque I'histoire est réalisée par la force de l'homme, la femme, la montre dans le domaine des apparences. Elle arrive ainsi à compléter ce qui lui manque naturellement. La surabondance des termes de la mode écrase «l'honnête homme à pied» (1793, p. 11).

\section{Conclusion}

La mode est un phénomène en perpétuelle mutation, une forme de fuite en avant, une quête toujours neuve et sans temps mort. Le règne de la mode illustre le culte de nouveauté et superficialité. Et c'est un lieu commun à l'auteur de Comment m'habillerai-je? de freiner le phénomène en proposant un projet contre l'excès des apparences. Mercier s'est appuyé sur la physiologie naturelle (médecins et philosophes des Lumières) et la littérature utopique (Bougainville), qui préconisent d'avantage la simplicité comme signe de beauté, santé, spontanéité, transparence. Littérature, philosophie et médecine ont croisé leurs approches afin de naturaliser à l'extrême la féminité : " constitution délicate », " tendresse excessive », "nerfs fragiles », etc. ; I'accent est mis sur l'infériorité intellectuelle et physiologique de la femme. La cause des femmes est loin d'être soutenue. La nature humaine est pensée comme une norme esthétique, morale et sociale. Comment m'habillerai-je ? est un projet qui s'enracine dans la tyrannie du grand goût classique et la belle nature. Ces idées avaient, semble-t-il, séduit Napoléon. Pour relancer les industries mises à mal par la révolution, l'Empereur avait imposé le retour au costume de cour selon le principe de l'Ancien régime. Pour les hommes, obligation de porter un uniforme basé sur le classique habit à la française, utilisant le velours ou le satin de soie brodé. Pour les femmes, des robes de cour en satin de soie brodées d'or ou d'argent avec des manteaux également brodés.

\section{Bibliographie}

MERCIER DE COMPIÈGNE Claude, Comment m'habillerai-je ? Réflexions politiques et philosophiques sur l'habillement français, et sur la nécessité d'un costume national, Paris, de l'imprimerie de l'auteur, 1793.

\section{ÉTUDES CRITIQUES}

BARTHES Roland, "Histoire et sociologie du vêtement », Annales Économies-Sociétés-Civilisations, vol. 12, N³, Paris, Armand Colin, 1957, p. 430-441.

CLINTON Katherine, "Femme et philosophe: Enlightenment origins of feminism », Eighteenth Century Studies, vol. 8, N³, 1975, p. 283-299.

DE LA BRETONNE Rétif, Les Nuits de Paris : La semaine nocturne, vingt nuits de Paris, [1788-1794], Le Plan-de-la-Tour, éd. d'Aujourd'hui, 1978. 
DELAPIERRE Madeleine, Se vêtir au XVIIIe siècle, Paris, Adam Brio, 1996.

DEVOCELLE Jean-Marc, " D'un costume politique à une politique du costume. Approches théoriques et idéologiques du costume pendant la Révolution française », in Modes et Révolutions 1780-1804, Paris, Paris-Musées, 1989, p. 83-103. FORTASSIER Rose, Les écrivains français et la mode de Balzac à nos jours, Paris, PUF, 1988. HOFFMANN Paul, La femme dans la pensée des Lumières, Paris, Ophrys, 1977.

KLEINERT Annemarie, "La mode, miroir de la Révolution française », in Modes et Révolutions 1780-1804, Paris, éd. Paris-Musées, 1989, p. 59-81.

KNIBIEHLER Yvonne, "Les médecins des Lumières et la nature féminine », in E. Morin-Rotureau (dir.), 1789-1799: combats de femmes, la Révolution exclut les citoyennes, Paris, Autrement, 2003, p. 127-142.

L'ISLE-ADAM Villiers (de), L'Ėve future, édition établie par Pierre Citron, Lausanne, L'Age d'Homme, coll. «Romantiques », 1979.

PERROT Philippe, Le travail des apparences ou les transformations du corps féminin XVIII XIXe siècle, Paris, Seuil, 1984.

SÉGUY Philippe, Histoire des modes sous I'Empire, Paris, Tallandier, 1988.

VITTU Françoise, "1780-1804 ou vingt ans de Révolution des têtes françaises », in Modes et Révolutions 1780-1804, Paris, éd. Paris-Musées, 1989, p. 41-57.

Recebido em: 15/08/2015 Aceito em: 06/11/2015

Referência eletrônica: KAHIA, Béchir. Mercier de Compiègne et la question de la mode. Criação \& Crítica, n. 15, p. 74-86, dez. 2015. Disponível em: <http://revistas.usp.br/ criacaoecritica>. Acesso em: dd mmm. aaaa.

Mercier de Compiègne et la question de la mode 